\title{
Medical Image of the Week: Idiopathic Pulmonary Hemosiderosis
}

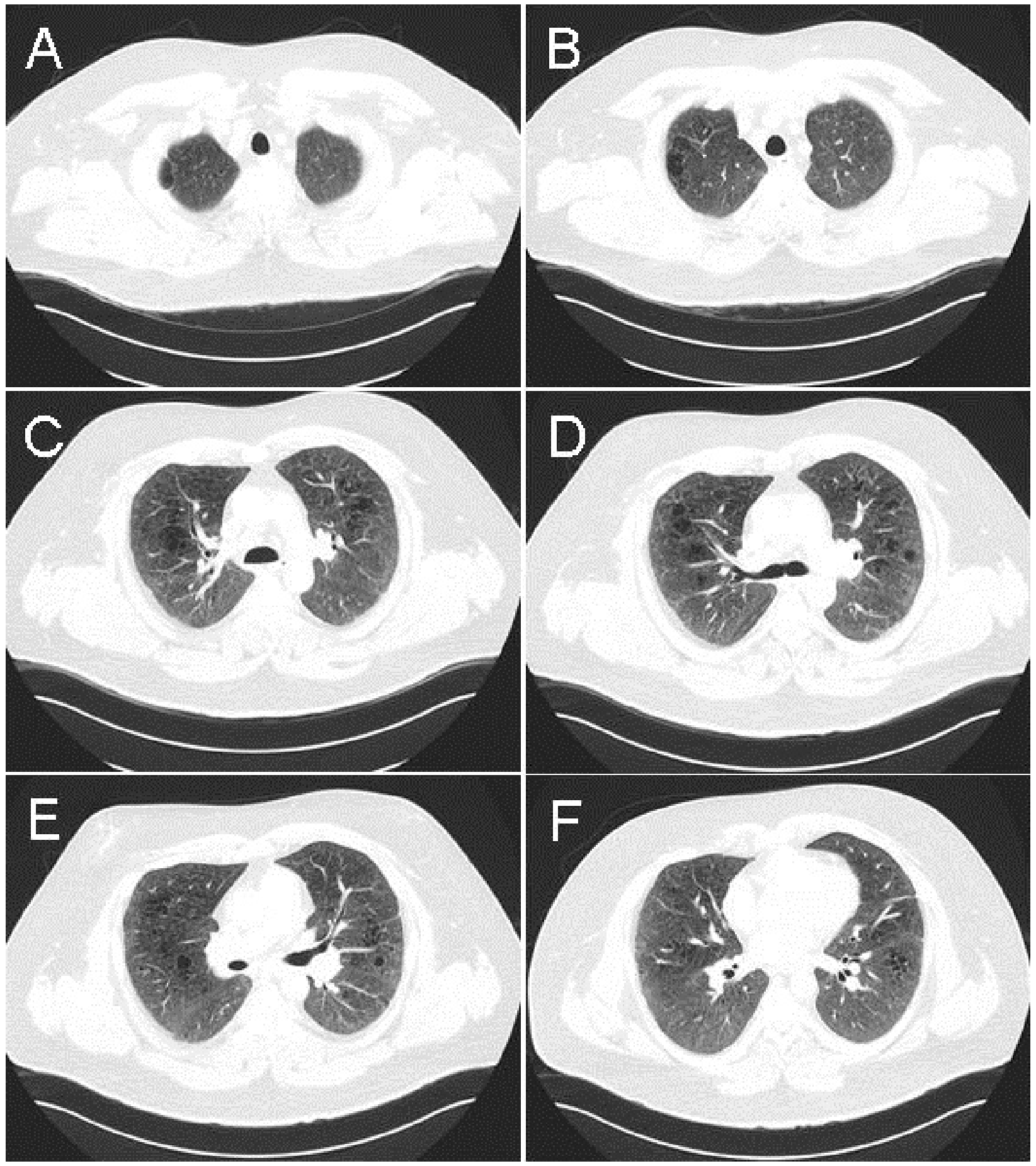

Figure 1. Representative axial high-resolution CT (HRCT) scan sections demonstrating increased attenuation of the lungs due to diffuse groundglass opacification with subpleural and scattered clustered cysts most evident in the upper lung zones. 
The patient is a 40 year-old man who was diagnosed with Idiopathic Pulmonary Hemosiderosis (IPH) at the age of three. He has recurrent episodes of hemoptysis several times a year that are controlled with increased doses of prednisone. He is chronically on $10 \mathrm{mg}$ daily which usually control his symptoms. A HRCT scan of the chest shows predominantly upper lung cystic changes both subpleural and clustered with a honeycomb appearance superimposed on a background of diffuse ground glass opacification.

Typical HRCT findings include patchy scattered areas of ground glass opacity and consolidation that usually involve the perihilar and lower aspects of the lungs. However, case reports of rare findings of multiple honeycomb cystic changes have been reported that are thought to be a result of progressive fibrotic changes from hemosiderin deposition in the interstitium (1). These honeycomb cysts may represent sites of more severe and recurrent alveolar hemorrhage in adults with IPH and are probably related to a traction phenomenon secondary to interstitial fibrosis following recurrent episodes of alveolar hemorrhage.

Nathaniel Reyes MD*, Linda Snyder MD*, Veronica Arteaga $\mathrm{MD}^{+}$ Department of Medicine, Division of Pulmonary and Critical Care Medicine* Department of Radiology ${ }^{+}$ University of Arizona, Tucson, Arizona

\section{Reference}

1. Harte S, McNicholas WT, Donnelly SC, Dodd JD. Honeycomb cysts in idiopathic pulmonary haemosiderosis: high-resolution CT appearances in two adults. $\mathrm{Br} \mathrm{J}$ Radiol. 2008;81(972):e295-8. [CrossRef] [PubMed] 